\title{
2020 Zuckerkandl Prize
}

\author{
David A. Liberles ${ }^{1}$
}

๑) Springer Science+Business Media, LLC, part of Springer Nature 2021

This year, 2020, the year of Covid (2019-nCoV), marks the second year in a row that the reinstated Zuckerkandl Prize has been awarded. This is an award for the top paper published in Journal of Molecular Evolution to appear in a print issue during the calendar year, as judged by a committee, in honor of Founding Editor Emile Zuckerkandl. The award committee consisting of Erich Bornberg-Bauer (University of Muenster, Germany), Joana Carneiro da Silva (University of Maryland School of Medicine, USA), and Ashley Teufel (Santa Fe Institute, USA) was assembled and the entire editorial board was polled for nominations to the committee.

Among the papers that were nominated, two received serious discussion from the committee. The runners up paper is listed below and reflects an important advance in molecular evolution.

- Sydykova et al., "Moderate Amounts of Epistasis are Not Evolutionarily Stable in Small Populations" (Sydykova et al. 2020).

In fact, this particular paper was the subject of a perspectives that was commissioned after its acceptance.

- McCandlish and Lang, "Evolution of Epistasis: Small Populations Go Their Separate Ways" (McCandlish and Lang 2020).

I am pleased to announce that "The Coevolution of Cellularity and Metabolism Following the Origin of Life" by Takagi et al. (2020) is the Zuckerkandl Prize Award winner for 2020. This study examines the conditions under which cellularity and an embedded metabolism emerge using a novel and simple, but biologically justified simulation scheme. Without aiming to do so, the work is concordant with existing theory on when cellularity is likely to emerge (Szathmáry 2007) in finding that the lack of environmental availability of compounds that can be processed to generate energy drives the selective advantage for the emergence of cellularity with a corresponding metabolism to generate energy. As such, this is an important contribution to origins of life research. Journal of Molecular Evolution is proud to congratulate the authors on this work.

\section{References}

McCandlish DM, Lang GI (2020) Evolution of epistasis: small populations go their separate ways. J Mol Evol 88:418-420. https://doi. org/10.1007/s00239-020-09950-4

Sydykova DK, LaBar T, Adami C, Wilke CO (2020) Moderate amounts of epistasis are not evolutionarily stable in small populations. $\mathbf{J}$ Mol Evol 88:435-444. https://doi.org/10.1007/s00239-020-09942 $-4$

Szathmáry E (2007) Coevolution of metabolic networks and membranes: the scenario of progressive sequestration. Philos Trans R Soc B 362:1781-1787

Takagi YA, Nguyen DH, Wexler TB, Goldman AD (2020) The coevolution of cellularity and metabolism following the origin of life. J Mol Evol 88:598-617. https://doi.org/10.1007/s00239-020-09961 $-1$

David A. Liberles

daliberles@temple.edu

1 Department of Biology and Center for Computational Genetics and Genomics, Temple University, Philadelphia, PA 19122, USA 